\title{
REDSHIFT DISTRIBUTION OF QUASI-STELLAR OBJECTS AND THE RADIO SOURCE COUNTS
}

\author{
VAHÉ PETROSIAN
}

Stanford University, Stanford, Calif., U.S.A.

\begin{abstract}
The evolution of QSOs and the reality of their apparent cutoff for $z>2.3$ is determined using their large scale redshift distribution. The contributions of QSRs to radio source counts are calculated on the basis of possible evolution of QSOs having $z>2.2$.
\end{abstract}

We discuss the large scale redshift distribution of QSOs to determine their evolution and the reality of the apparent cutoff in their distribution for redshift $z>2.3$, in the relativistic cosmological model with zero cosmological constant and with deceleration parameter $q_{0}=+1$. We shall not discuss the distribution in the Lemaître models which were introduced in 1967 (Petrosian et al.) and have been reviewed recently (Petrosian

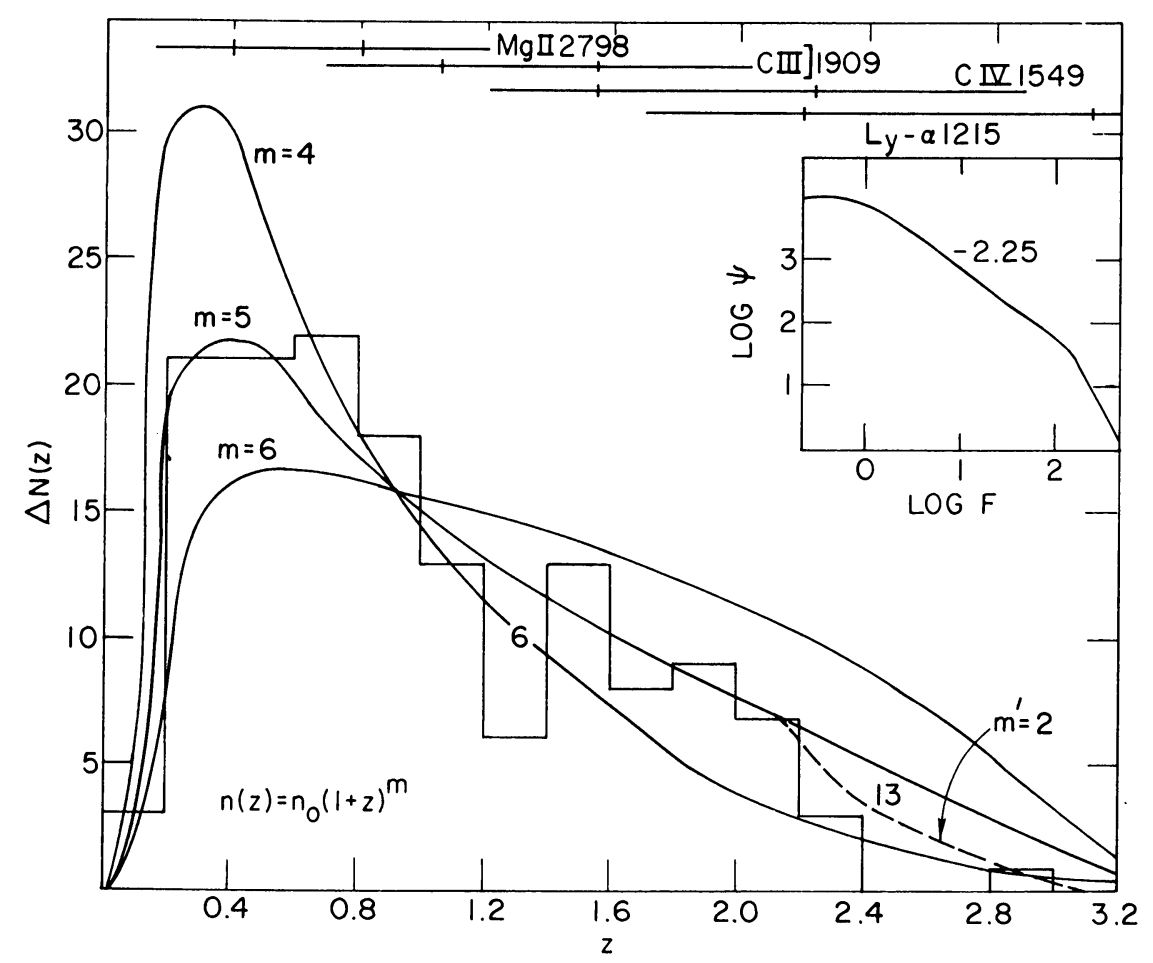

Fig. 1. Redshift distribution of QSRs. The numbers indicate source deficiencies. The upper right hand corner curve is the radio luminosity function with the indicated logarithmic slope. The horizontal lines show the range of redshifts where the various strong lines appear in the $\mathrm{U}, \mathrm{B}, \mathrm{V}$ bands. The lack of sources at $z \approx 1.3$ may be attributed to the absence of two strong and reliable lines around this redshift (Petrosian, 1970). 


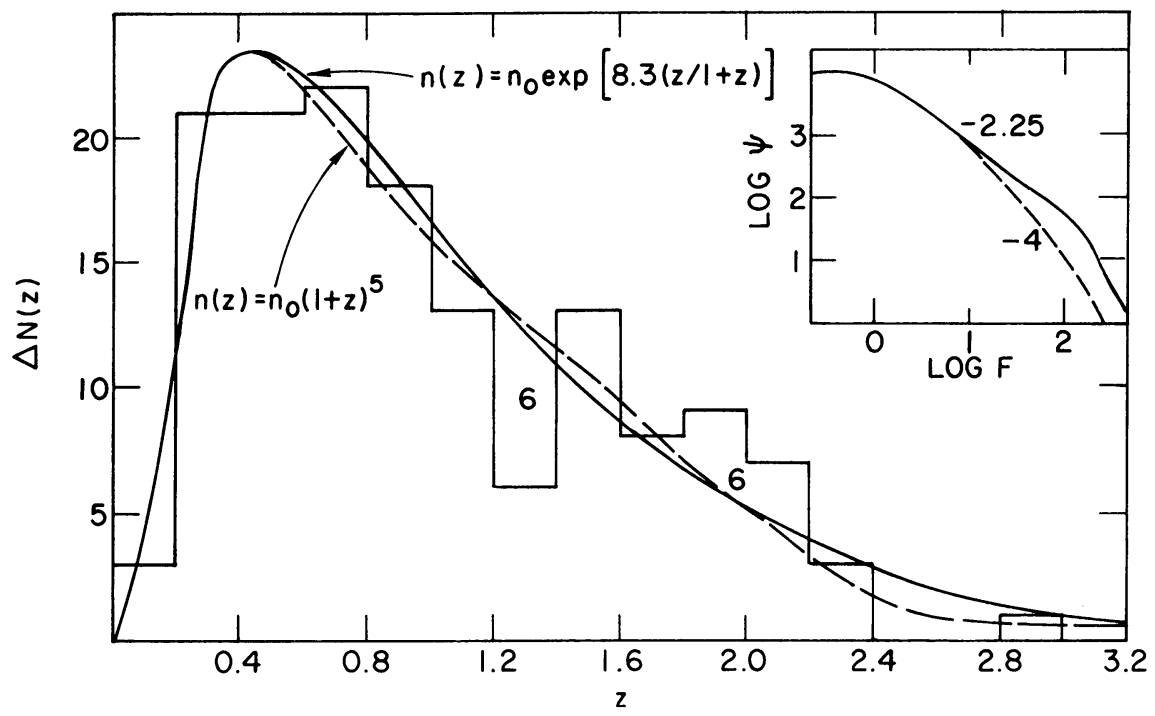

Fig. 2. Redshift distribution of QSRs. The numbers indicate source deficiencies. The upper right hand corner curve is the radio luminosity function with the indicated logarithmic slopes.

and Salpeter, 1970). In a recent article (Petrosian, 1970) we have shown that the density evolution derived by Schmidt (1968) from the data on 33 3CR quasi-stellar radio sources (QSRs) cannot continue beyond $z>2.2$. In this report we discuss possible evolution of sources for $z>2.2$, then based on these models we calculate the contribution of QSRs to the radio source counts.

In Figures 1 and 2 we compare calculated distributions for various forms of the density evolution with the observed histogram. The calculations leading to these results and the optical luminosity function are discussed in detail in the above mentioned article (Petrosian, 1970) and will not be described here. On the upper right hand corner of Figures 1 and 2 we show the radio luminosity function. The curves in Figure 1 plotted for a power law density evolution $n(z)=n_{0}(1+z)^{m}$ show the possible range of the exponent $m$. The best fit is obtained for $m=5$. If this evolution were to continue beyond $z \approx 2.2$ we should have seen 13 sources at $z>2.2$. Only 4 sources are observed. This discrepancy can be eliminated if for $z>2.2, n(z)=$ $N_{0}(3.2)^{5} \times[(1+z) / 3.2]^{m^{\prime}}$ for $0<m^{\prime}<2$. The dashed curve in Figure 1 is for $m^{\prime}=2$. In Figure 2 we have plotted the expected distribution for an exponential density evolution (here the coefficient 8.3 of the exponent was chosen so that $n(2)=n_{0} \times 3^{5}$ ) and for a density evolution with a steeper radio luminosity function at large intrinsic radio luminosities (dashed curves). For these evolution laws there are fewer (4 and 2 respectively) missing sources at $z>2.2$, but there are 6 more sources than expected at $1.8<z<2.2$. These however, are within the expected statistical fluctuations (cf. also figure caption). As we shall see later these models might be ruled out by the lack of observed 'radio quiet' QSOs with $z>2.2$. Note that the dashed luminosity function has 0.4 times as many bright sources as the luminosity function obtained from the 
3CR sample by Schmidt (1968) (solid curve). This model can also be ruled out because it gives rise to an unacceptably large radio background.

We now discuss the implications of these evolution laws for the radio source counts. Based on these models we can calculate the number of sources up to any limiting radio flux density and optical magnitude. Table I shows some of these results for two power law density evolution with $m=5, m^{\prime}=0$ and $m=5.5, m^{\prime}=2$. The first three rows give

TABLE I

$N(\mathrm{QSR}) /$ sterradian

\begin{tabular}{|c|c|c|c|c|c|c|c|c|c|}
\hline \multirow[b]{2}{*}{$\begin{array}{l}\text { Optical } \\
\text { limit }\end{array}$} & \multirow[t]{2}{*}{$\begin{array}{l}\text { Radio } \\
\text { limit }\end{array}$} & \multicolumn{2}{|l|}{$3 \mathrm{CR}$} & \multicolumn{2}{|l|}{ Parkes } & \multicolumn{2}{|l|}{$4 \mathrm{C}$} & \multicolumn{2}{|c|}{ Zero flux density } \\
\hline & & $\begin{array}{l}m=5 \\
m^{\prime}=0\end{array}$ & $\begin{array}{l}m=5.5 \\
m^{\prime}=2\end{array}$ & $\begin{array}{l}m=5 \\
m^{\prime}=0\end{array}$ & $\begin{array}{l}m=5.5 \\
m^{\prime}=2\end{array}$ & $\begin{array}{l}m=5 \\
m^{\prime}=0\end{array}$ & $\begin{array}{l}m=5.5 \\
m^{\prime}=2\end{array}$ & $\begin{array}{l}m=5 \\
m^{\prime}=2\end{array}$ & $\begin{array}{l}m=5.5 \\
m^{\prime}=2\end{array}$ \\
\hline $18^{m} \cdot 4$ & & $6.1(33)$ & $6.1(33)$ & 10 & 10 & 38 & 41 & 870 & 1200 \\
\hline $19^{m} .0$ & & $7.7(42)$ & $8.1(44)$ & 13 & 15 & 51 & 58 & 1600 & 2700 \\
\hline $20^{m} \cdot 0$ & & $9.3(50)$ & $11.5(62)$ & 18 & 21.2 & 73 & 93 & 2500 & 8300 \\
\hline Total & & $13(70)$ & $15(81)$ & 25 & 30 & 100 & 150 & 8700 & 27000 \\
\hline Radio $\mathrm{g}$ & alaxies & 30 & 28 & 80 & 75 & 520 & 470 & & \\
\hline
\end{tabular}

the number of QSRs per steradian (the numbers in parenthesis give the number of QSRs normalized to 33 sources seen up to $18^{m} 4$ ) up to visual magnitudes $18.4,19.0$ and 20.0 for flux limits of the $3 \mathrm{C}$, Parkes, and the $4 \mathrm{C}$ surveys and to zero limiting flux density. The fourth line gives the total number of QSRs per steradian expected in these surveys. We note first that these results agree approximately with the identification of 53 QSRs in the 3CR survey (Wyndham, 1966) and about 90 QSRs per steradian in the 4C catalogue (Olsen, 1970), which indicates that only a few of the unidentified sources can be'QSRs (cf. also Bolton, 1969). If we subtract the numbers in the 4th row from the observed number of radio sources we obtain the number per steradian of radio galaxies (5th row). The comparison of the numbers in the last two rows shows that although the QSRs obey the same density evolution law as is required for the powerful radio sources (Longair, 1966), they cannot be the only cause of the observed steep $\log N-\log S$ relations. Therefore, the radio galaxies must also evolve as indicated previously (Rowan-Robinson, 1967; Petrosian, 1969).

Based on these models we can also calculate the redshift distribution of the quasistellar radio sources which are absent from the radio catalogues (because of their low intrinsic radio luminosities and large redshifts) but which are optically bright enough to be seen on the sky surveys ('radio quiet' QSOs). This distribution and the histogram of the observed 'radio quiet' QSOs are shown in the lower portion of Figure 3, which shows a large discrepancy between these distributions. Since the sample of the observed sources is small and has been obtained by various observers with a variety of techniques this discrepancy might be due to fluctuations or selection effects other than those selection effects included in our calculation. However, the presence of 
a large number of observed QSOs with $z<0.4$ is not compatible with the observed distribution of the QSRs. Furthermore, the average absolute visual magnitude of these sources is $3 \mathrm{mag}$ larger than that of the sources in this histogram with $z>1.0$ or the average absolute visual magnitude of the QSRs. Attempts (Lang and Terzian,
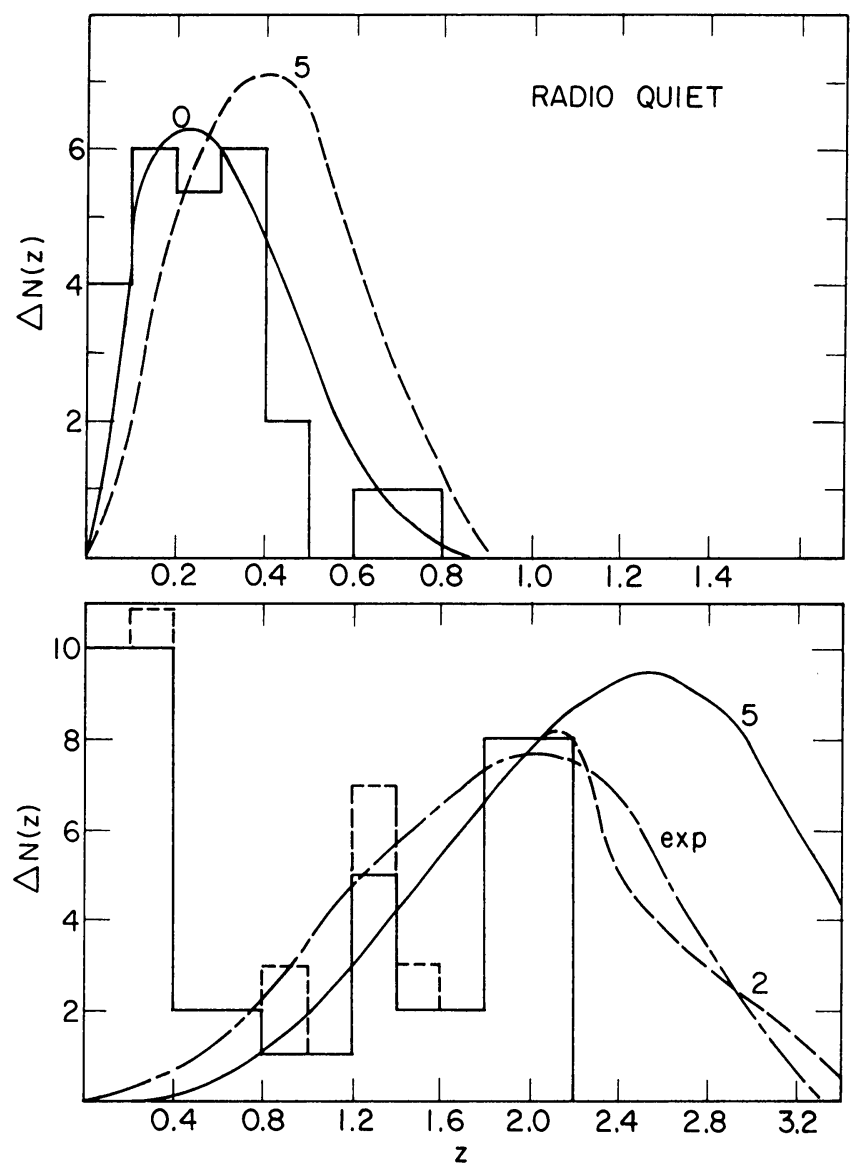

Fig. 3. Redshift distribution of 'radio quiet' QSOs. The curves in the lower portion are for QSRs missing from the radio surveys for various evolution laws. The remainder of the histogram is plotted on the upper part in an expanded scale with the calculated curves for no evolution (solid curve) and with power law evolution $n(z)=n_{0}(1+z)^{5}$ (dashed curve).

1968; Grueff, 1970) to detect radio emission from the sources with $z<0.4$ indicate that their radio emission is comparable with or less than the radio emission of weak radio galaxies and ordinary galaxies. This difference leads us to speculate that most of the large redshift objects in this histogram are QSRs missing from the radio catalogue while the low redshift objects which have low optical luminosity and are extremely weak radio emitters are the only truly radio quiet QSOs. If these speculations are correct then the space density of the radio quiet QSOs must be 50 (roughly 
$0.5 \times$ (volume up to $z=2) /($ volume up to $z=0.4)$ ) times larger than previously estimated. If we then normalize the observed and the calculated distributions at $z \approx 2$, as has been shown in Figure 3, we note that: (i) the observed distribution indicates a more severe cutoff at $z>2.2$ than obtained from the evolution laws of the QSRs; (ii) as shown on the top portion of Figure 3 the solid curve distribution obtained for a flat luminosity function and no evolution agrees better with the histogram of the low $z$ radio quiet QSOs than the dashed curve drawn for a power law evolution $n(z)=n_{0}(1+z)^{5}$. These results are quite tentative and the suggested distribution of the 'radio quiet' QSOs should not be taken seriously. We mention these possibilities here to indicate that the so called 'radio quiet' QSOs might not constitute a homogeneous set of sources. This fact should be kept in mind in their statistical analysis.

\section{References}

Bolton, J. G.: 1969, Astron. J. 74, 131.

Grueff, G.: 1970, Astrophys. J. Letters 160, L41.

Lang, K. R. and Terzian, Y.: 1968, Astrophys. J. Letters 158, L11.

Longair, M. S.: 1966, Monthly Notices Roy. Astron. Soc. 133, 421.

Olsen, E. T.: 1970, preprint, Owens Valley Radio Observatory, California Institute of Technology,

Pasadena, California.

Petrosian, V.: 1969, Astrophys. J. 155, 1029.

Petrosian, V.: 1970, Astrophys. Letters 6, 71.

Petrosian, V. and Salpeter, E. E.: 1970, Comments Astrophys. Space Sci. 2, 109.

Petrosian, V., Salpeter, E. E., and Szekeres, P.: 1967, Astrophys. J. 147, 1222.

Rowan-Robinson, M.: 1967, Nature 216, 1289.

Schmidt, M.: 1968, Astrophys. J. 151, 393.

Wyndham, J. D.: 1966, Astrophys. J. 144, 459.

\section{Discussion on Papers Read by Rowan-Robinson and Petrosian}

McVittie: I should like to remark that Richard Ringenberg and I have solved the integral equation needed to determine the density evolution function in radio source counts from the counts themselves for a number of cosmological models. The number of sources rises to a maximum for large redshifts and then falls off. Thus no cutoffs are needed in this kind of evolution.

Ekers: I wish to make a cautionary remark regarding the identification of galaxies as faint as magnitude 20. Especially for extended sources when we cannot rely heavily on positional agreement, there is no way to judge the validity of an identification. It does seem likely from the radio source counts that the radio galaxies have the same evolutionary properties as the QSOs but since the powerful radio emitters are as powerful as the QSOs this may be occurring at much greater redshifts than implied by our analysis.

Rowan-Robinson: You would have to say that all the identifications of $3 \mathrm{C}$ sources below $18^{\mathrm{m}} .5$ were wrong.

Rowan-Robinson (replying to Mackay): I accept that the estimated magnitudes provide a very unreliable indication of redshift, and I make a plea to observers to measure the redshifts of the $3 \mathrm{C}$ radio galaxies.

Miss Merkelijn: I have applied the volume-halves $\left(V / V_{m}\right)$ test to a complete sample of approximately one hundred radio galaxies obtained from identifications based on the Parkes catalogue.

The optical completeness limit of the sample lies at $m_{\mathrm{B}}=19$ and the radio completeness limit at $S_{400}=3.5 \mathrm{f}$.u. The rms error in the magnitudes is $0^{m} .6$. The results are in good agreement with those reported by Dr Rowan-Robinson.

Kafka: Perhaps the point raised by Dr Schmidt can be made still clearer. A luminosity function 
is determined by counting objects of some class of luminosity within some volume which is determined by the definition of completeness of the observed sample. If two different criteria are used for this definition, the usual concept of a luminosity function no longer makes much sense. For the sample used by Schmidt (1968) one should rather consider the 'two-dimensional' (joint radio and optical) luminosity function, that is a mountain over the $L_{\mathrm{rad}}-L_{\mathrm{opt}}$ plane. The sample does not allow us to determine the number per unit volume of QSS within some range of (say) $L_{\text {opt }}$. If the radio limit were decreased, this number, the integral over the mountain along the line of fixed $L_{\text {opt }}$, would grow considerably. Also there is no reason to expect that the shapes of the 'one-dimensional' luminosity functions remain unchanged if one integrates with respect to $L_{\mathrm{rad}}$ over the larger ranges of $L_{\mathrm{opt}}$ appearing in the plane, if different limits of completeness are used. It is a pity that one needs a much larger sample to determine the shape and height of the mountain which will contain a lot of information on individual and cosmological evolution. In the near future (years) there will be faster progress with the purely optical luminosity function determined from a sample picked only by optical criteria.

Petrosian: (written reply to a remark by Dr Schmidt). The calculation described should be correct as long as the sample of $333 \mathrm{CR}$ sources used for luminosity function determination is not a fluctuation but a representative sample. In fact the new luminosity function obtained by Dr Schmidt (paper submitted to Astrophys. $J$.) does not change our calculations. The new total luminosity function is

$$
\phi(L, F)=\phi_{\mathrm{opt}}(L) G\left(\frac{F}{L}\right) \propto L^{-1.4}\left(\frac{F}{-}\right)^{-1.0}=L^{-0.4} F^{-1.0},
$$

where $\phi$ 's refer to number of sources within a logarithmic interval of radio and optical luminosities $F$

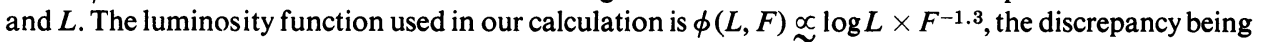
due to small sample of sources and the fact that two different methods were used for determining the luminosity function (in fact we have used various luminosity functions including $\phi(L, F) \propto L^{-0.3} F^{-1.0}$ without noticing a substantial change in our conclusions). Thus, this new result (that the optical luminosity function of the sample chosen by their optical properties alone is steeper than the optical luminosity function of $3 \mathrm{CR}$ quasi-stellar sources) substantiates what we have said about the sample of 'radio quiet' sources. Whether this is due to the difference between radio quiet and radio active sources or due to an optical luminosity dependent radio luminosity function must await the radio observation of the new sample. 